\title{
Clinical implications of adiponectin and inflammatory biomarkers in type 2 diabetes mellitus
}

\author{
Menha Swellam ${ }^{\mathrm{a}, *}$, Magda Sayed Mahmoud ${ }^{\mathrm{a}}$ and Adel Abdel-Fatah Ali ${ }^{\mathrm{b}}$ \\ ${ }^{\mathrm{a}}$ Department of Biochemistry, Genetic Engineering and Biotechnology Research Division, National Research \\ Center, Dokki, Giza, Egypt \\ ${ }^{\mathrm{b}}$ Internal Medicine Department, Faculty of Medicine, Alazhar University, Naser City, Cairo, Egypt
}

\begin{abstract}
Objectives: To study the interrelationships of adiponectin, C-reactive protein (CRP) and fibrinogen with each other in T2DM patients with (T2DM-C) and without complications (T2DM-NC) among healthy individuals.

Design and methods: The study comprised of 120 T2DM-C, 59 T2DM-NC patients and 40 healthy volunteers. Biochemical markers were determined in the serum.

Results: Positivity rates of CRP and fibrinogen were significantly increased in T2DM-C as compared to T2DM-NC or controls, whereas adiponectin showed highest level in healthy individuals. Inflammatory biomarkers were inversely correlated with adiponectin $(P<0.01)$. Lipid profiles, kidney functions and BMI, showed positive significant correlation with CRP and fibrinogen but negative correlation with adiponectin. For better detection of T2DM, the combined sensitivity (98.9\%) and specificity (92.5\%) of fibrinogen and adiponectin was higher than the combined sensitivity and specificity of fibrinogen and CRP or adiponectin and CRP or than that of the biomarkers alone.

Conclusion: Elevated levels of CRP and fibrinogen and reduced level of adiponectin can be used for early diagnosis of T2DM and can predict diabetic complications.
\end{abstract}

Keywords: Type 2 diabetes mellitus, adiponectin, c-reactive protein, fibrinogen

\section{Introduction}

The epidemic proportion of people with diabetes is alarming [1]. Type 2 diabetes affects small (microangiopathy) or large vessels (macroangiopathy). Microvascular disease is the hallmark of retinopathy, neuropathy, and nephropathy, whereas macroangiopathy in diabetes is manifested by accelerated atherosclerosis, which affects vital organs (heart and brain) [2]. Therefore, attention has been focused recently on whether

\footnotetext{
* Corresponding author: Associate Prof. Dr. Menha Swellam, Biochemistry Department, Genetic Engineering and Biotechnology Research Division, National Research Center, Tahrir Street, Dokki, Giza, 12622, Egypt. Tel.: +20 23335451; Fax: +20 23370931; E-mails: menha_m_swellam@hotmail.com, menha_m_swellam@ yahoo.com.
}

the unconventional risk factors are independently associated with vascular risk, and if they provide clinically significant prognostic value beyond the established ones [3].

Adiponectin is an adipocyte-derived peptide with evidence of diabetic and cardio-protective properties, with low circulating levels in the obese [4] and in metabolic disorders associated with increased fat mass including hyperglycemia, insulin resistance, dyslipidemia, and vascular inflammation [5].

An accumulating body of evidence suggests that inflammation may play a crucial intermediary role in pathogenesis, thereby linking diabetes with a number of commonly coexisting conditions thought to originate through inflammatory mechanisms [6]. In this regard, substantial experimental evidence and more recent cross-sectional data suggest that C-reactive pro- 
tein (CRP), a sensitive physiological marker of subclinical systemic inflammation, is associated with hyperglycemia, insulin resistance, and overt T2DM [7]. A study by Takebayashi and colleagues suggested that CRP appeared not to be a discriminatory factor for diabetic complications, although it probably had a pathogenetic role at the level of the vascular endothelium. The role of CRP in diabetic patients in regards to obtaining "value-added" information is therefore, still controversial [8]. Also, Fibrinogen; a plasma protein produced in liver, is regulated by cytokines and is greatly enhanced by the acute phase response to inflammatory processes [9]. It contributes more than other proteins to plasma viscosity in healthy subjects. This contribution is greatly increased in disease states, among them diabetes mellitus. Elevated plasma viscosity is the feature of diabetic blood, which results in greater flow resistance, and a high incidence of certain complications. Long before the biochemical deviation in carbohydrate metabolism is demonstrable, changes occur in the small blood vessels due to elevated viscosity of plasma that are responsible for some of the complications associated with the disease [10].

When T2DM is clinically diagnosed there may already be diabetic complications, [11]. These clinical findings have lead clinicians to the hypothesis that polygenic T2DM is a vascular disease rooted in endothelial genetic defects and occurs as a result of interactions with environmental stressors such as over nutrition, obesity, and under exercise in the metabolic syndrome with hyperglycemia being a late manifestation [12].

The aim of the present study was to explore the association among CRP, fibrinogen and adiponectin in T2DM with and without complications to evaluate their usefulness as screening biomarkers for early prediction of complications in patients with T2DM.

\section{Materials and methods}

\subsection{Study population}

This cohort study included 179 with confirmed diagnosis as type 2 diabetes mellitus (T2DM) at the time of blood draw. Based on revised American Diabetes Association diagnostic criteria [13], cases were confirmed if one or more of the following conditions were met: (1) presence of more than 1 classic symptom of hyperglycemia (i.e. polyuria, polydipsia, weight loss with or without polyphagia, and blurred vision) plus either a fasting plasma glucose of $126 \mathrm{mg} / \mathrm{dL}$
(7.0 $\mathrm{mmol} / \mathrm{L})$ or higher or random plasma glucose $200 \mathrm{mg} / \mathrm{dL}$ (11.1 mmol/L) or higher; (2) in the absence of symptoms, 2 or more elevated plasma glucose concentrations (fasting plasma glucose of $\geqslant 126 \mathrm{mg} / \mathrm{dL}$ $[7.0 \mathrm{mmol} / \mathrm{L}]$, random plasma glucose $\geqslant 200 \mathrm{mg} / \mathrm{dL}$ [11.1 mmol/L], or 2-hour plasma glucose $\geqslant 200 \mathrm{mg} / \mathrm{dL}$ [11.1 mmol/L] during oral glucose tolerance testing); or (3) use of insulin or an oral hypoglycemic agent. Of the diabetic participants; 59 candidates were diabetic mellitus type II without complications, and they were 36 females (mean age $47.4 \pm 9.2$ years; range 2959 years) and 23 males (mean age $54 \pm 9.3$ years; range 35-63 years) with BMI (BMI; calculated as weight in kilograms divided by the square of square of height in meters) ranged from $20-35 \mathrm{Kg} / \mathrm{m}^{2}$. Because therapeutic approaches are often very different in the management of patients with diabetes and this significantly influences their vascular complications, we reasoned to include in the present study only subjects not previously treated with vascular medications and with a first diagnosis of type 2 diabetes, thus the remaining 120 diabetic mellitus type 2 candidates were newly diagnosed with diabetic vascular complications [cardiovascular disease $97(80.8 \%)$ such as ischemic heart diseases, hypertension and myocardial infraction.; nephropathy $76(63.3 \%)$; retinopathy $71(59.2 \%)$ and neuropathy $110(91.7 \%)]$, they were examined with Echo-Color Doppler, opthalmoscopic and fluoroangiography, also urinary albumin excretion (UAE) from 24-h urine collection was determined using radial immunodiffusion assay, neuropathy was diagnosed by the presence of abnormal sensation in the extremities. They were categorized according to the gender status into 63 females (mean age $54.2 \pm 8$ years; range $43-70$ years) and 57 males (mean age $55.8 \pm 8.3$ years; range $43-81$ years) with BMI ranged from $23.8-39 \mathrm{Kg} / \mathrm{m}^{2}$. A group of clinically healthy volunteers $(n=40)$ were enrolled in the study to serve as control group and include $26 \mathrm{fe}$ males (mean age $49.5 \pm 7.5$ years; range $30-59$ years) and 14 males (mean age $51.3 \pm 13$ years; range 2963 years) with BMI ranged from $20-29 \mathrm{Kg} / \mathrm{m}^{2}$.

\subsection{Blood collection and processing}

The study was approved by the ethical committee of the University of Alzhraa hospital, and informed consent was obtained from all participants. Venous Blood samples were withdrawn at enrollment after overnight fasting; each sample was divided into three portions: the first was mixed gently with $150 \mu$ l EDTA solution then divided into two tubes; one was centrifuged for 
15 min. at 3000 RPM., the supernatant (plasma) was stored in liquid nitrogen until laboratory assay for CRP and adiponectin, the other tube is used immediately to measure glycosylated hemoglobin (Hb)A1c. The second portion was added to $0.2 \mathrm{ml} \mathrm{Na}$ citrate $3.2 \%$ and mixed gently, then centrifuged and the plasma was separated for immediate estimation of plasma fibrinogen. The third portion was left to clot at room temperature and serum was separated after centrifugation for assessment of lipid profile, kidney function and serum glucose.

Plasma C-reactive protein (CRP) was measured using the US CRP ELISA kit (Diagnostic Systems Laboratories, Inc., Webster, TX) [14]. Plasma adiponectin concentrations were measured by ELISA kit (R\&D Systems, Minneapolis, MN) [15]. Hemoglobin $(\mathrm{Hb}) \mathrm{A} 1 \mathrm{c}$ was measured by chromatographic spectro-photometric ion exchange resin using kit supplied by Biosystems company (S.A, Barcelona, Spain). Assessment of plasma fibrinogen was carried out by modification of the method on system CA analyzer (HITACHII-911TM Roche Diagnostic System, Boheringer Manheim) [16]. Measurement of lipid profile, kidney function and serum glucose was carried out photo-metrically using HITACII-911TM Autoanalyzer (Roche Diagnostic System, Boheringer Manheim).

\subsection{Statistical analysis}

The threshold value for optimal sensitivity and specificity was determined by receiver operating characteristics (ROC) curve which was constructed by calculating the true-positive fraction (sensitivity \%) and falsepositive fraction (100-specificity \%) of CRP, fibrinogen, and adiponectin at several cutoff points [17]. The Kruskal-Wallis and Mann-Whitney $U$ non-parametric tests were used for the statistical comparison of the variables between the various groups. The chi-square test was utilized to compare the positivity rates and to study the association between the different variables. Pearson's correlations were used to test the relationship between variables. The level of significance was determined to be less than 0.05 . All analyses were performed using the Statistical Package for the Social Sciences (SPSS software, version 12, Chicago, Illinois).

\section{Results}

\subsection{Clinical characteristics of the study cohort}

The clinical characteristics of the controls, diabetic mellitus type 2 with (T2DM-C) and, without (T2DM-
NC) complications are shown in (Table 1). Significant difference was observed between all the clinical characteristics among the three investigated groups as well as between T2DM-C as compared to either T2DM-NC or the control groups $(P<0.0001)$. The mean levels of CRP and fibrinogen, were increased 2.07 fold, and 1.28 fold respectively in T2DM-C group as compared to T2DM-NC group and 4.04 fold and 2.5 fold, respectively in T2DM-C group as compared to control group $(P<0.0001)$. While the mean level of CRP and fibrinogen were increased 1.95 fold and 1.97 fold respectively in T2DM-NC group as compared to control group $(P<0.0001)$. On the other hand, adiponectin level was increased 4.45 fold in control group compared to T2DM-C group and 2.6 fold compared to T2DM-NC group. In T2DM-NC group, adiponectin levels were higher 1.69 fold than T2DM-C group $(P<0.0001)$.

\subsection{Correlation between biomarkers in type 2 diabetes mellitus group}

To assess the associations between biomarkers in T2DM, we calculated Pearson correlation coefficients. Significant positive associations were reported between $\mathrm{CRP}$, fibrinogen and $(\mathrm{Hb}) \mathrm{A} 1_{\mathrm{c}}(R=0.398,0.163)$, LDL $(R=0.228,0.22)$, triglyceride $(R=0.278$, $0.329)$, urea $(R=0.194,0.349)$, creatinine $(R=0.22$, $0.242)$, fasting $(R=0.419,0.338)$ and postprandial glucose ( $R=0.391,0.398)$, and BMI ( $R=0.294$, 0.256 ) respectively at $P<0.001$, while negative association was detected between CRP, fibrinogen and HDL $(R=-0.305,-0.15$ at $P<0.001$, respectively). For adiponectin, negative associations were detected between adiponectin and $(\mathrm{Hb}) \mathrm{A} 1 \mathrm{c}, \mathrm{LDL}$, triglyceride, creatinine, fasting and postprandial glucose, and $\operatorname{BMI}(R=-0.275,-0.231,-0.309,-0.174,-0.173$, -0.235 , and -0.282 respectively at $P<0.02$ ), while positive association was detected between adiponectin and HDL ( $R=0.186, P=0.013)$. Moreover, CRP was positively associated with fibrinogen and negatively associated with adiponectin (Fig. 1A-C).

\subsection{Positivity rates of CRP, adiponectin, and fibrinogen among different investigated groups}

Best cutoff values for CRP, fibrinogen and adiponectin, were calculated using ROC curve as shown in Fig. 2. There was statistical difference in the rates of aforementioned biomarkers among the three investigated groups, Table 2. 
Table 1

Characteristics by mean $(95 \% \mathrm{CI})$ for the investigated biomarkers among the different studied groups

\begin{tabular}{|c|c|c|c|}
\hline Biomarkers & Control & T2DM-NCII & T2DM-C $\#$ \\
\hline $\mathrm{CRP}(\mathrm{mg} / \mathrm{L})$ & $0.75(0.5-0.9)$ & $1.46(1.3-1.6)^{\mathrm{c}}$ & $3.03(2.78-3.28)^{\mathrm{a}, \mathrm{b}, \mathrm{d}, \mathrm{e}}$ \\
\hline Fibrinogen $(\mathrm{mg} / \mathrm{dl})$ & $182(168-195)$ & $359(330-353)^{\mathrm{c}}$ & $461(427-495)^{\mathrm{a}, \mathrm{bd}, \mathrm{e}}$ \\
\hline Adiponectin $(\mu \mathrm{g} / \mathrm{ml})$ & $32.9(27.8-38)$ & $12.5(10.3-14.7)^{\mathrm{c}}$ & $7.4(6.44-8.44)^{\mathrm{a}, \mathrm{b}, \mathrm{d}, \mathrm{e}}$ \\
\hline $\mathrm{GlyHb}(\mathrm{Hb}) \mathrm{A} 1 \mathrm{c} \%$ & $5.3(5-5.6)$ & $7(6.4-7.4)^{\mathrm{c}}$ & $10.8(10.57-11.1)^{\mathrm{a}, \mathrm{b}, \mathrm{d}, \mathrm{e}}$ \\
\hline Cholesterol (mg/dl) & $155(147-163)$ & $188(179-197)^{\mathrm{c}}$ & $191(184-197)^{\mathrm{a}, \mathrm{b}, \mathrm{d}}$ \\
\hline HDL-C (mg/dl) & $50.1(47-51.7)$ & $45.24(43-47)^{\mathrm{C}}$ & $33.6(32.3-34.8)^{\mathrm{a}, \mathrm{bd}, \mathrm{e}}$ \\
\hline $\mathrm{LDL}-\mathrm{C}(\mathrm{mg} / \mathrm{dl})$ & $81(74-88)$ & $80(75-86)$ & $131(108-119)^{\mathrm{a}, \mathrm{bd}, \mathrm{e}}$ \\
\hline Triglyceride (mg/dl) & $106(94-118)$ & $107(97-116)$ & $174(166-188)^{\mathrm{a}, \mathrm{bd}, \mathrm{e}}$ \\
\hline Urea (mg/dl) & $30(28-31)$ & $29(28-31)$ & $37(33-40)^{\mathrm{a}, \mathrm{d}, \mathrm{e}}$ \\
\hline Creatinine (mg/dl) & $0.85(0.8-0.9)$ & $0.85(0.8-0.89)$ & $1.1(1-1.2)^{\mathrm{a}, \mathrm{b}, \mathrm{d}, \mathrm{e}}$ \\
\hline FBS (mg/dl) & $86.6(84-89)$ & $86(84-88$ & $211(201-222)^{a, b, d, e}$ \\
\hline PPS (mg/dl) & $99(95-102)$ & $98.8(96-101)$ & $301(283-318)^{a, b, d, e}$ \\
\hline BMI $\left(\mathrm{kg} / \mathrm{m}^{2}\right)$ & $22.1(21.3-22.9)$ & $30.17(29.5-31)^{\mathrm{c}}$ & $33.27(32.8-33.7)^{\mathrm{a}, \mathrm{b}, \mathrm{d}, \mathrm{e}}$ \\
\hline Systolic BP (mmHg) & $124(115-120)$ & $130(120-132)$ & $140(125-145)^{\mathrm{a}, \mathrm{b}, \mathrm{d}, \mathrm{e}}$ \\
\hline Diastolic BP (mmHg) & $75(70-77)$ & $84(72-88)$ & $101(80-104)^{a, b, d, e}$ \\
\hline
\end{tabular}

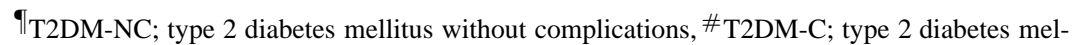
litus with complications. Significant $P<0.0001$ between, ${ }^{\text {a } a l l ~ s t u d i e d ~ g r o u p s ~ a t ~} P<0.0001$ using non-parametric analysis (Kruskal-Wallis), ${ }^{\mathrm{b}} \mathrm{T} 2 \mathrm{DM}$ and control groups, ${ }^{\mathrm{c}} \mathrm{T} 2 \mathrm{DM}-\mathrm{NC}$ and control groups, ${ }^{\mathrm{d}} \mathrm{T} 2 \mathrm{DM}-\mathrm{C}$ and control groups, ${ }^{\mathrm{e}} \mathrm{T} 2 \mathrm{DM}-\mathrm{C}$ and T2DM-NC groups, and using non-parametric analysis (Mann - Whitney test).

Table 2

Distributions of CRP, adiponectin and fibrinogen among different investigated groups using the cutoff values

\begin{tabular}{|c|c|c|c|c|c|c|}
\hline \multirow{2}{*}{$\begin{array}{l}\text { Investigated } \\
\text { groups (no.) }\end{array}$} & \multicolumn{2}{|c|}{$\mathrm{CRP}^{*}$} & \multicolumn{2}{|c|}{ Fibrinogen* } & \multicolumn{2}{|c|}{ Adiponectin* } \\
\hline & $\leqslant 1.07 \mathrm{mg} / \mathrm{L}$ & $>1.07 \mathrm{mg} / \mathrm{L}$ & $\leqslant 242 \mathrm{mg} / \mathrm{dl}$ & $>242 \mathrm{mg} / \mathrm{dl}$ & $\leqslant 14 \mu \mathrm{g} / . \mathrm{ml}$ & $>14 \mu \mathrm{g} / . \mathrm{ml}$ \\
\hline Control & $32(80 \%)$ & $8(20 \%)$ & $38(95 \%)$ & $2(5 \%)$ & $6(15 \%)$ & $34(85 \%)$ \\
\hline T2DM-NC & $18(30.5 \%)$ & $41(69.5 \%)$ & $3(5.1 \%)$ & $56(94.9 \%)$ & $40(67.8 \%)$ & $19(32.2 \%)$ \\
\hline T2DM-C & $6(5 \%)$ & $114(95 \%)$ & $8(6.7 \%)$ & $112(93.3 \%)$ & $105(87.5 \%)$ & $15(12.5 \%)$ \\
\hline
\end{tabular}

* Significant $P<0.0001$ using Pearson Chi-square test.

\subsection{Adiponectin, CRP and fibrinogen among T2DM-C group}

According to the studied T2DM patients, $67 \%$ (120/179) patients showed complications categorized as follows; $80.8 \%(97 / 120)$ with cardiovascular diseases, $63.3 \%(76 / 120)$ with nephropathy, $91.7 \%$ (110/120) with neuropathy, and 59.2\% (71/120) with retinopathy. As indicated in Table 3 , the three biomarkers of interest were significantly associated with CVD, and nephropathy. Moreover, CRP was associated with retinopathy and adiponectin was associated with neuropathy.

The median percentage change for CRP and fibrinogen in T2DM-C group revealed positive association with different complications. On the other hand, adiponectin revealed negative association with different complications (Fig. 3). Among the entire group of T2DMC (Table 3), CRP was increased in T2DM patients with CVD, nephropathy and retinopathy. On the other hand, adiponectin was decreased in T2DM patients with CVD, nephropathy and neuropathy. Fibrinogen showed significant increment in T2DM patients with CVD and nephropathy.

\subsection{Combined sensitivity and specificity of investigated biomarkers}

Sensitivity and specificity for CRP, fibrinogen and adiponectin as well as their combination were tested for detection of T2DM and T2DM-C, as shown in Table 4. Both sensitivity (93\%) and specificity (95\%) of fibrinogen were the highest among the other two biomarkers for detection of T2DM. Absolute sensitivity was reached when the three biomarkers were combined although the specificity was decreased. For detection of complications in T2DM patients, CRP revealed the highest sensitivity (95\%) and specificity $(50.5 \%)$ as compared with adiponectin and fibrinogen. Absolute sensitivity was reached when CRP was combined with adiponectin, although the specificity was reduced to $42.9 \%$. 


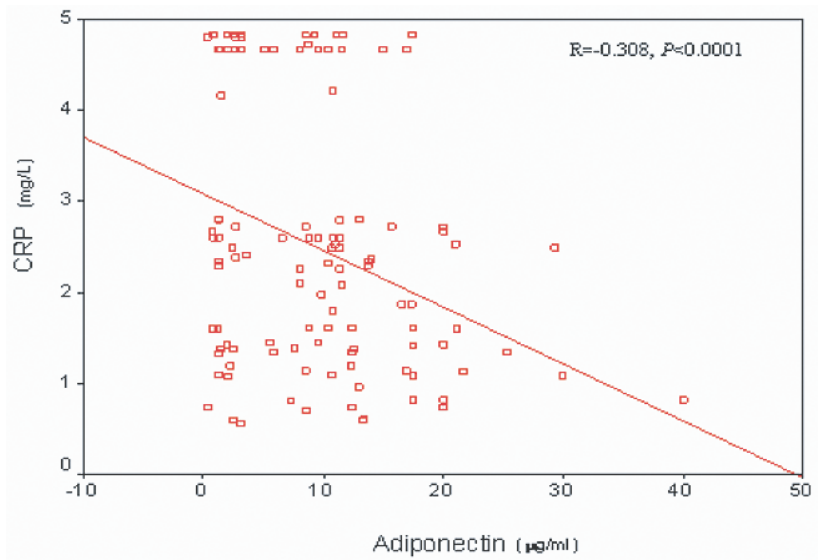

(a)

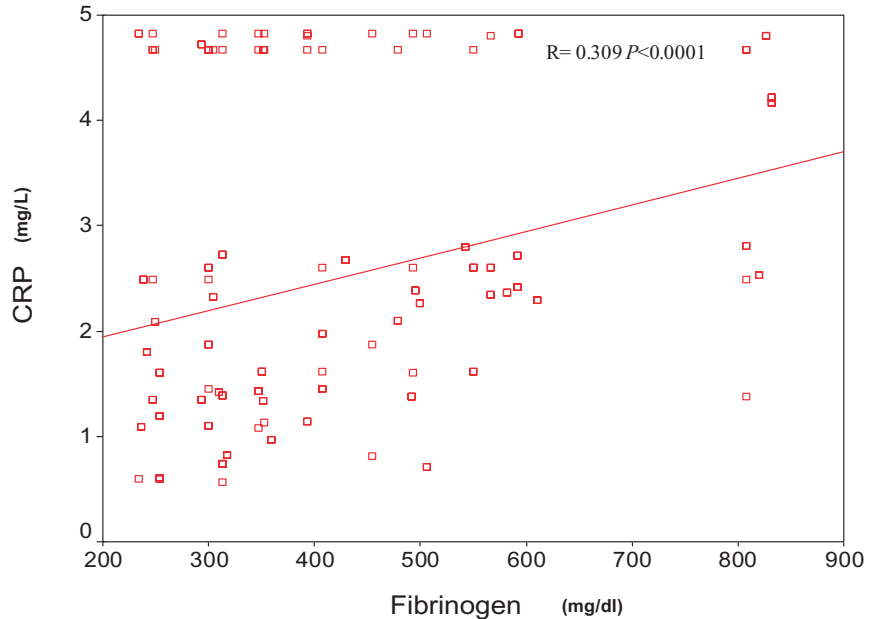

(b)

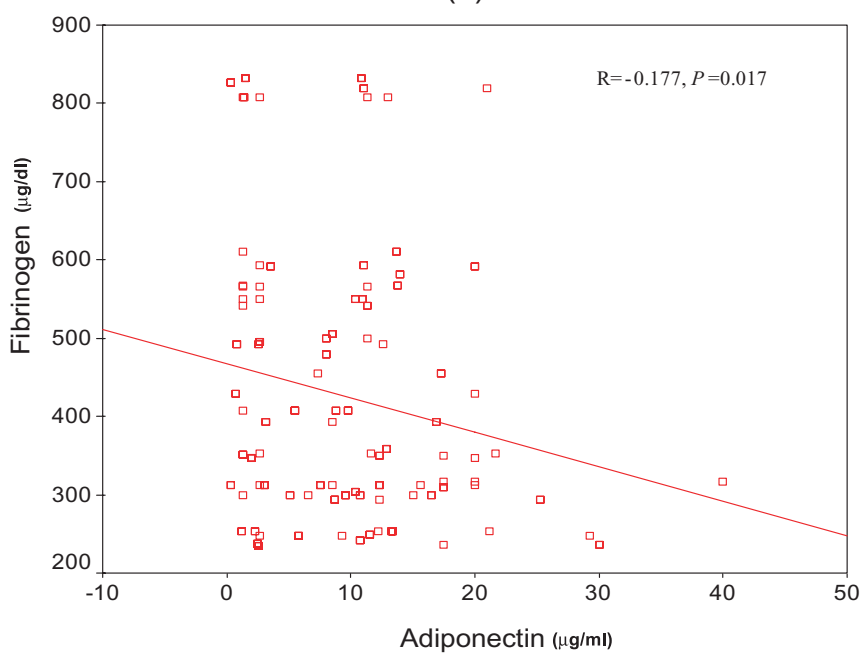

(c)

Fig. 1. Linear regression analysis between (1a) CRP and adiponectin, (1b) CRP and fibrinogen and (1c) adiponectin and fibrinogen among T2DM group. 


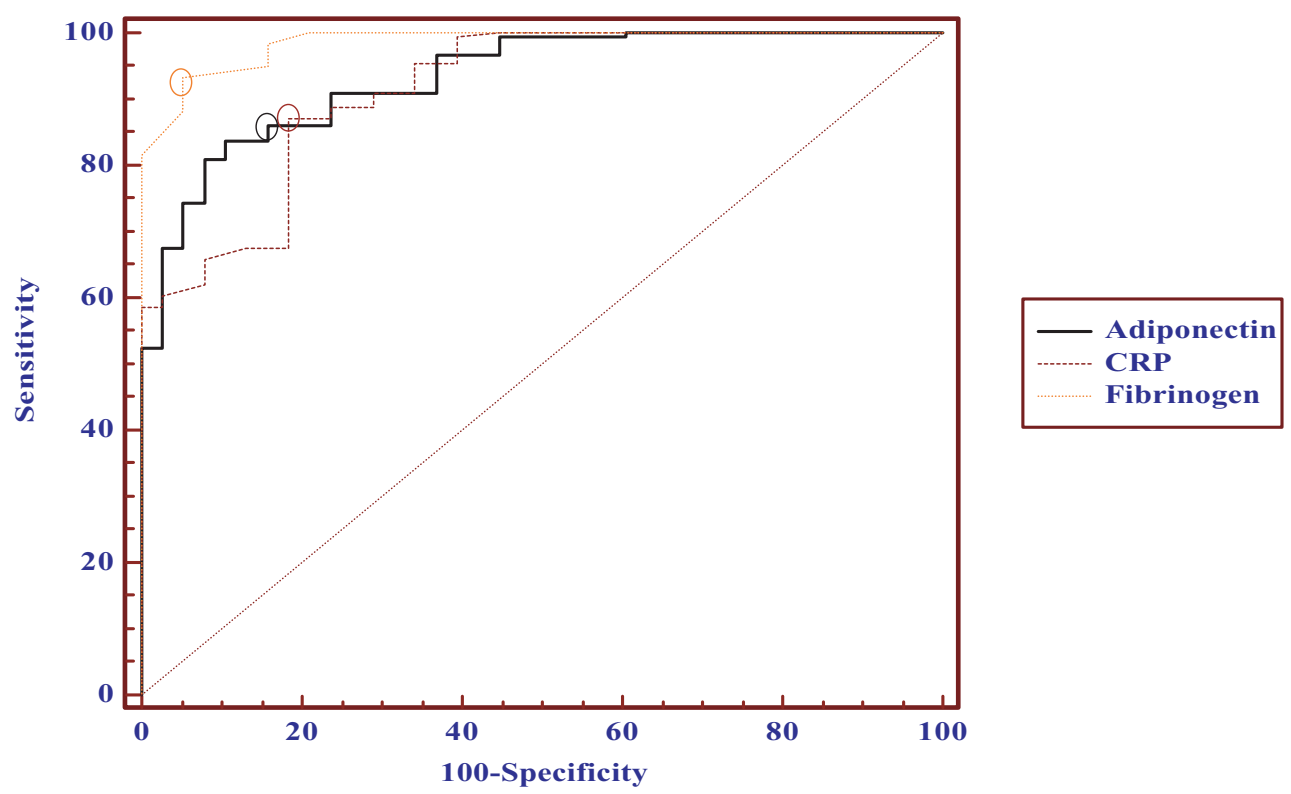

Fig. 2. ROC curve analysis for CRP, fibrinogen and adiponectin to calculate the best cutoff point to discriminate between control and diabetic groups. Open circles denote best cutoff points of CRP (straight line) as $1.07 \mathrm{mg} / \mathrm{L}$ [sensitivity $=86.6 \%$ and specificity $=80 \%$. Area under the curve (AUC) $[\mathrm{SE}]=0.871[0.034], 95 \%$ confidence limits range $=0.805-0.937, P<0.0001]$, fibrinogen as $242 \mathrm{mg} / \mathrm{dl}$ [sensitivity $=93.9 \%$ and specificity $=95 \%$. Area under the curve $(\mathrm{AUC})[\mathrm{SE}]=0.986[0.007], 95 \%$ confidence limits range $=0.973-0.999, P<0.0001]$ and adiponectin as $14(\mu \mathrm{g} / \mathrm{ml})$ [sensitivity $=81 \%$ and specificity $=85 \%$. Area under the curve (AUC) [SE] $=0.922[0.022]$, 95\% confidence limits range $=0.879-0.964, P<0.0001]$.

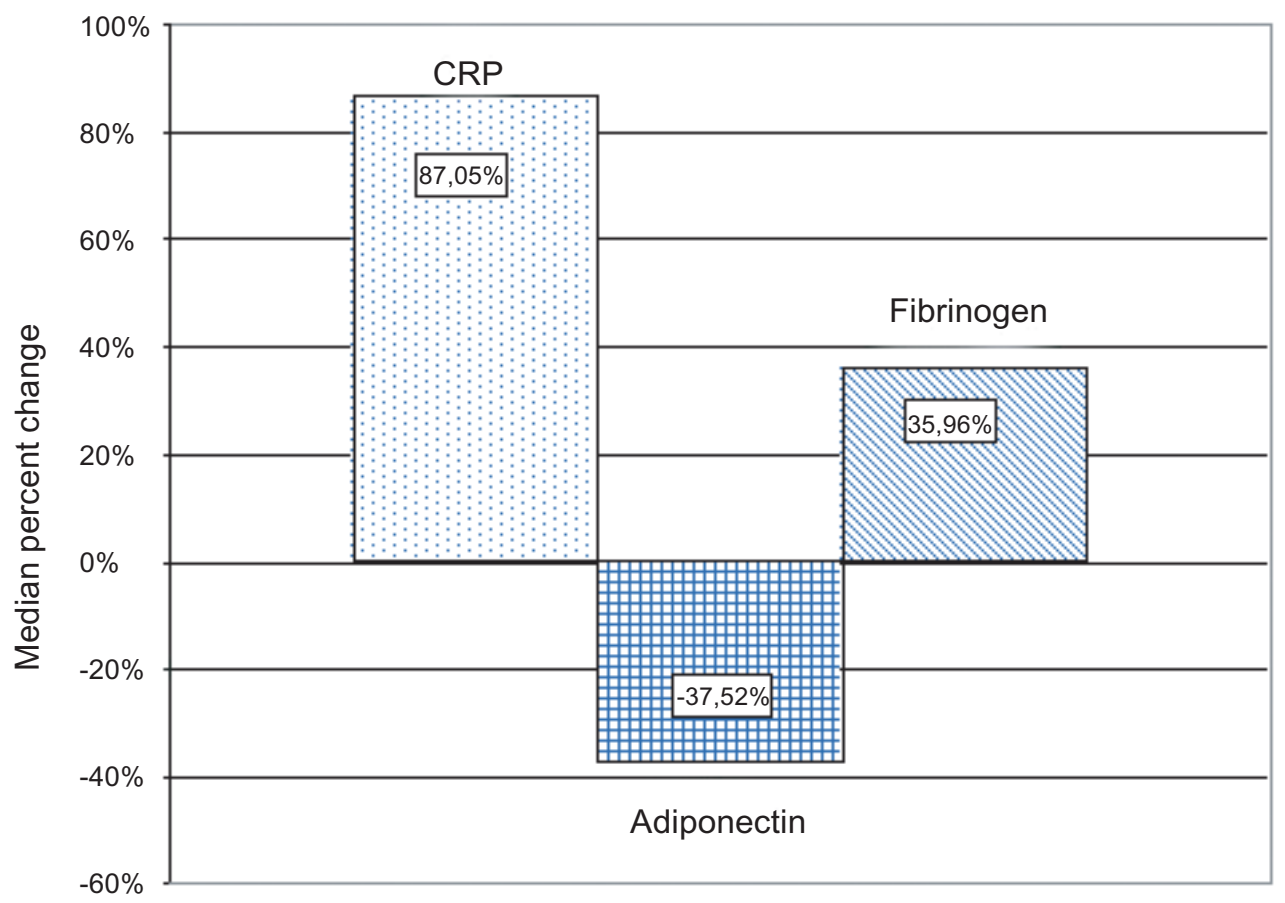

Fig. 3. The median percentage change of the CRP, fibrinogen and adiponectin among the T2DMC as controled for T2DM-NC. 
Table 3

Distribution of complications in T2DM-C group among the CRP, fibrinogen and adiponectin

\begin{tabular}{|c|c|c|c|c|c|c|}
\hline \multirow{2}{*}{ Complications } & \multicolumn{2}{|c|}{$\mathrm{CRP}(\mathrm{mg} / \mathrm{L})$} & \multicolumn{2}{|c|}{ Fibrinogen $(\mathrm{mg} / \mathrm{dl})$} & \multicolumn{2}{|c|}{ Adiponectin $(\mu \mathrm{g} / \mathrm{ml})$} \\
\hline & $>1.07$ & Mean & $>242$ & Mean & $\leqslant 14$ & Mean \\
\hline \multicolumn{7}{|l|}{ CVD } \\
\hline Negative & $17(14.9 \%)^{\mathrm{a}}$ & $2.26^{\mathrm{b}}$ & $19(17 \%)^{\mathrm{a}}$ & $341.8^{\mathrm{b}}$ & $15(14.3 \%)^{\mathrm{a}}$ & $9.82^{\mathrm{b}}$ \\
\hline Positive & $97(85.1 \%)$ & 3.22 & $93(83 \%)$ & 489.5 & $90(85.7 \%)$ & 6.88 \\
\hline \multicolumn{7}{|l|}{ Nephropathy } \\
\hline Negative & $39(34.2 \%)^{\mathrm{a}}$ & $2.56^{\mathrm{b}}$ & $37(33 \%)^{\mathrm{a}}$ & $396.3^{\mathrm{b}}$ & $34(32.4 \%)^{\mathrm{a}}$ & $8.83^{\mathrm{b}}$ \\
\hline Positive & $75(65.8 \%)$ & 3.31 & $75(67 \%)$ & 498.7 & $71(67.6 \%)$ & 6.64 \\
\hline \multicolumn{7}{|l|}{ Neuropathy } \\
\hline Negative & $10(8.8 \%)$ & 2.91 & $10(8.9 \%)$ & 463.4 & $4(3.8 \%)^{\mathrm{a}}$ & $11.47^{\mathrm{b}}$ \\
\hline Positive & $104(91.2 \%)$ & 3.04 & $102(91.1 \%)$ & 461 & $101(96.2 \%)$ & 7.07 \\
\hline \multicolumn{7}{|l|}{ Retinopathy } \\
\hline Negative & $44(38.6 \%)^{\mathrm{a}}$ & $2.67^{\mathrm{b}}$ & $45(40.2 \%)$ & 463 & $41(39 \%)$ & 7.07 \\
\hline Positive & $70(61.4 \%)$ & 3.28 & $67(59.8 \%))$ & 458.6 & $64(61 \%)$ & 8 \\
\hline
\end{tabular}

Significant $P<0.001$ using ${ }^{\text {a }}$ Pearson Chi-square test, and ${ }^{\mathrm{b}}$ ANOVA test.

Table 4

Combined sensitivity and specificity of CRP, fibrinogin and adiponectin in T2DM, especially T2DM with complications

\begin{tabular}{lccccc}
\hline \multirow{2}{*}{ Biomarkers } & \multicolumn{2}{c}{ Detection of T2DM } & & \multicolumn{2}{c}{ Detection of T2DM-C } \\
\cline { 2 - 3 } \cline { 5 - 6 } & Sensitivity & Specificity & & Sensitivity & Specificity \\
\hline CRP & 86 & 80 & & 95 & 50.5 \\
Fibringen & 93 & 95 & & 93.3 & 41.4 \\
Adiponectin & 81 & 85 & & 87.5 & 53.5 \\
CRP + Fibringen & 98.3 & 80 & & 99 & 34.3 \\
CRP + Adiponectin & 98.1 & 87.1 & & 100 & 42.9 \\
Fibringen + Adiponectin & 98.9 & 92.5 & & 100 & 35.4 \\
CRP + Adiponectin + Fibringen & 100 & 67.5 & & 100 & 27.3 \\
\hline
\end{tabular}

\section{Discussion}

Diabetes mellitus type 2 (T2DM) is a complex disease with altered expression of many biomarkers, which can be anticipated to interact. It is therefore critical to monitor their changes and their inter-relationships with other risk factors [1].

In the present study, we performed quantitative analysis of CRP, fibrinogen and adiponectin in serum samples from different investigated groups by ELISA to evaluate their usefulness in diagnosis of T2DM as well as early screening of complications associated with T2DM. Accordingly, the levels and positivity (sensitivity) of both inflammatory markers (CRP and fibrinogen) were significantly increased in T2DM-C as compared to T2DM-NC and controls as reported earlier by others [7]. On the other hand, our results were in concordance with Mantozoros and his colleagues [18] as the levels and positivity rates of the circulating adiponectin were lower in T2DM-C as compared to T2DM-NC and controls $(P<0.0001)$. These results indicate that elevation of inflammatory markers and reduced adiponectin levels play an important role not only in the pathophysiology of T2DM but also in the category of complications associated with this type of disease.
Authors investigated the correlation between the aforementioned biomarkers and glycosylated hemoglobin $(\mathrm{Hb}) \mathrm{A} 1 \mathrm{c}$, lipid profile, kidney function and BMI. Inflammatory markers were positively correlated with glycosylated hemoglobin (Hb)A1c, fasting and postprandial glucose, LDL and triglyceride while negatively correlated with HDL. Gorink and his coworkers [19] reported that advanced glycation end products (AGEs) that formed by glycosylated hemoglobin are associated with pathogenesis of diabetic complications through interaction with their receptors (RAGE) which can induce numerous changes linked to inflammation. In addition, increased plasma glucose contributes to the hyperfibrinogenemia of type 2 diabetes [20]. Also, it was previously reported that serum HDL-cholesterol concentrations are associated with triglyceride-rich lipoprotein metabolism and body fat in young healthy men. Therefore, an inverse association between HDL-cholesterol and inflammatory markers found in the present study suggests that low HDL-cholesterol may be related to chronic low-grade inflammation, as well as subtle abnormalities in triglyceride-rich lipoprotein metabolism in young healthy men. Low HDL-cholesterol in low grade inflammation in the present study may be com- 
patible with the recent observation that human secretory phospholipase $\mathrm{A} 2$, an acute-phase protein, decreased HDL-cholesterol in response to inflammation [21]. Our results revealed strong correlation between the level of the inflammatory markers and BMI in T2DM patients as previously reported by Kazumi et al. [22] suggesting that obesity may be a state of low grade inflammation as well. This maybe attributed to the fact that increased synthesis of some cytokines in obese women lead to increased synthesis of acute - phase reactants like CRP in liver [23]. Adiponectin, a member of a new family of obesity-related hormones, the adipocytokines, which is produced solely by white adipose tissue [24], was negatively correlated with ( $\mathrm{Hb}) \mathrm{A} 1 \mathrm{c}$, fasting and postprandial glucose indicating as previously reported [18] that higher adiponectin levels are associated with better glycemic control. Moreover, it was negatively correlated with triglycerides, indicating that adiponectin may reduce intrahepatic and muscle triglyceride content through increased muscle fat oxidation and induction of genes which are important in fatty acid transport and oxidation. Also, its level was reversed to obesity as measured by BMI, this is may be due to some of the common polymorphisms in the promoter region, exon and intron 2 and the rare mutations in exon 3 of the human adiponectin gene [25]. However, adiponectin was positively correlated with HDL. These results are in line with report from von Eynatten et al. [26] who found an association between decreased post-heparin lipoprotein lipase (LPL) activity and low plasma adiponectin independent of systemic inflammation and insulin resistance. Conversely, dramatically raised levels of LPL activity have been found with increased plasma adiponectin in an animal model. Thus, adiponectin may directly stimulate the expression of LPL, which then will result in increased HDL-C levels.

T2DM is the leading cause of new cases of blindness, end-stage renal disease, and increased risk of cardiovascular disease. Most studies have indicated that increased risk for these complications can not be explained solely by conventional risk factors such as dyslipidemia, hypertension, and smoking. Therefore, the diabetic state, per se, confers an increased propensity to accelerated atherogenesis; however, the precise mechanisms by which this occurs remain to be elucidated. Regarding patients with type 2 diabetes, an increased incidence of cardiovascular diseases has been reported among those with elevated plasma levels for those markers of inflammation

Preliminarily, we found strong significant correlations between the positivity rates of CRP and fibrino- gen levels among T2DM-C especially those with CVD and nephropathy, while only CRP showed parallel association with retinopathy $(P<0.0001)$. These findings could point out, as previously reported, the role of increased inflammation in the pathogenesis of these vasculopathies, moreover, confirming that the role of these two markers of inflammation is complementary. On the contrary, adiponectin was decreased in T2DM patients with CVD, nephropathy and neuropathy $(P<$ 0.0001). This could be attributed to the presence of a region of chromosome $3 q$ contains a susceptibility locus for diabetic nephropathy in patients with type 2 diabetes, and one group evaluated 14 candidate genes on chromosome $\mathrm{q}$ and found the strongest linkage with a SNP for the promoter of adiponectin [27]. These findings indicate that adiponectin deficiency accentuates CVD and kidney diseases among T2DM which indicate that identification of low adiponectin levels identifies high risk populations [28]. Moreover, the elevated adiponectin levels were increased in T2DM patients with nephropathy as compared to T2DM-NC which may result from enhanced filtration through the damaged kidney [29].

In the current study, the three biomarkers of interest were correlated with each other indicating that adipose tissue synthesizes and secretes adiponectin and other cytokines. Increased synthesis of these cytokines in obese subjects leads to insulin resistance in muscle, increased synthesis of acute-phase reactants in the liver (CRP and fibrinogen), and/or activation of macrophages in atheromatous plaques, which leads to an increased incidence of vascular diseases [24]. Moreover, when the median percentage changes of the three biomarkers were controlled for T2DM-NC, CRP and fibrinogen were increased while adiponectin was decreased among those with complications. These results suggest that T2DM patients should be treated aggressively and followed closely to prevent future complications usually associated with T2DM.

The early detection of T2DM would certainly improve the diagnosis and screening of high-risk groups, such as patients with CVD, nephropathy, neuropathy and retinopathy. This study is among the first to use combine CRP, fibrinogen and adiponectin in a joint study as novel biomarkers for T2DM. Fibrinogen showed both highest sensitivity and specificity as compared to either CRP or adiponectin alone for diagnosing of T2DM. Among the several combinations, both the sensitivity $(98.9 \%)$ and specificity $(92.5 \%)$ of fibrinogen and adiponectin together were higher as compared to other combinations. Absolute sensitivity was report- 
ed when the three biomarkers were combined although the specificity was decreased $(67.5 \%)$. Intriguingly, absolute sensitivity with a specificity $(42.9 \%)$; closer to that reported for CRP $(50.5 \%)$, adiponectin $(53.5 \%)$ and fibrinogen $(41.4 \%)$ when tested alone, were observed when CRP and adiponectin where combined.

Because recent studies have addressed the importance of therapeutic modulation of these biomarkers in high-risk patients for the prevention of vascular events [14], it also cannot be excluded that an effective primary prevention on the categories of patients with T2DM may take into account the reduction of elevated CRP and fibrinogen as well as elevation of adiponectin levels, thus enable better management of patients. However, beyond the utility of these biomarkers in the prediction of complications in patients with newly diagnosed diabetes, further studies should focus on the prospective analysis of these assays in larger multicentric studies to evaluate the therapeutic implications of these biomarkers.

\section{References}

[1] R. Sicree, J. Shaw, P. Zimmet and R. Tapp, The global burden of diabetes, in: Diabetes atlas 2003 (2nd edR), D. Gan Ed., Brussels, Belgium' International Diabetes Federation, 2003.

[2] J. Calles-Escandon and M. Cipolla, Diabetes and endothelial dysfunction: A clinical perspective, Endocrine Reviews 22 (2001), 36-52.

[3] D. Streja, P. Cressey and S.W. Rabkin, Associations between inflammatory markers, traditional risk factors, and complications in patients with type 2 diabetes mellitus, $J$ Diabetes Complications 17 (2003), 120-127.

[4] L. Qi, J.B. Meigs, S. Liu, J.E. Manson, C. Mantzoros and F.B. Hu, Dietary fibers and glycemic load, obesity, and plasma adiponectin levels in women with type 2 diabetes, Diabetes Care 29 (2006), 1501-1505.

[5] S.D. Poppitt, G.F. Keogh, F.E. Lithander, Y. Wang, T.B. Mulvey, Y.-K. Chan, B.H. McArdle and G.J.S. Cooper, Postprandial response of adiponectin, interleukin-6, tumor necrosis Factor- $\alpha$ and C-reactive protein to a high-fat dietary load, $\mathrm{Nu}$ trition 24 (2008), 322-329.

[6] A.D. Pradhan, J.E. Manson, N. Rifai, J.E. Buring and P.M. Ridker, C-reactive protein, Interleukin 6, and risk of developing type 2 diabetes mellitus, JAMA 286 (2001), 327-334.

[7] E. Corrado, M. Rizzo, I. Muratori, G. Coppola and S. Novo, Association of elevated fibrinogen and C-reactive protein levels with carotid lesions in patients with newly diagnosed hypertension or type II diabetes, Archives of Medical Res $\mathbf{3 7}$ (2006), 1004-1009.

[8] K. Takebayashi, M. Suetsugu, R. Matsutomo, S. Wakabayashi, Y. Aso and T. Inukai, Correlation of high-sensitivity C-reactive protein and plasma fibrinogen with individual complications in patients with type 2 diabetes, South Med J 99 (2006), 23-27.

[9] M. Javorsky, M. Kozarova, J. Salagovic and T. Kac, Relationship among urinary albumin excretion rate, lipoprotein lipase PvuII polymorphism and plasma fibrinogen in type 2 diabetic patients, Physiol Res 55 (2006), 55-2.
[10] M.D. Vestra, M. Mussap, P. Gallina, M. Bruseghin, A.M. Cernigoi, A. Saller, M. Plebani and P. Fioretto, Acute-phase markers of inflammation and glomerular structure in patients with type 2 diabetes, J Am Soc Nephrol 16 (2005), S78-2.

[11] Z. Bloomgarden, European Association for the Study of Diabetes (EASD). Meeting, Diabetic Care 25 (2002), 1229-6.

[12] M.R. Hayden, J.R. Sowers and S.C. Tyagi, The central role of vascular extracellular matrix and basement membrane remodeling in metabolic syndrome and type 2 diabetes: the matrix preloaded, Cardiovascular Diabetology 4 (2005), 1-20.

[13] Report of the Expert Committee on the Diagnosis and Classification of Diabetes Mellitus, Diabetes Care 20 (1997), 1183 1197.

[14] E. Lopez-Garcia, M.B. Schulze, T.T. Fung, J.B. Meigs, N. Rifai, J.E. Manson and F.B. Hu, Major dietary patterns are related to plasma concentrations of markers of inflammation and endothelial dysfunction, Am J Clin Nutr 80 (2004), 10291035.

[15] A.H. Berg, T.P. Combs and P.E. Schever, ACRP30/ Adiponectin: An adiponectin regulating glucose and lipid metabolism trends. Endocrinol, Meta 13 (2002), 84-85.

[16] N.S. Cooks and D. Ubben, Fibrinogen as a major risk factor in cardiovascular disease, T PS 11 (1990), 444-451.

[17] C.E. Metz, Basic principles of ROC analysis, Semin Nucl Med 8 (1978), 283.

[18] C.S. Mantzoros, T. Li, J.E. Manson, J.B. Meigs and F.B. $\mathrm{Hu}$, Circulating adiponectin levels are Associated with better glycemic control, more favorable lipid profile, and reduced inflammation in women with type 2 diabetes, $J$ Clin Endocrin \& Metab 90 (2005), 4542-4548.

[19] I. Gornik, O. Gornik and V. Gasparovic, HbAlc is outcome predictor in diabetic patients with sepsis, Diabetes Res and Clin Practice 77 (2007), 120-125.

[20] R. Barazzoni, M. Zanetti, G. Davanzo, E. Kiwanuka and P. Carraro, Increased fibrinogen production in type 2 diabetic patients without detectable vascular complications: Correlation with plasma glucagon concentrations.

[21] U.J. Tietge, C. Maugeais and S. Land-Katz, Human secretory phospholipase A2 mediates decreased plasma levels of HDL cholesterol and Apo A-I in response to inflammation in human apo A-I transgenic mice, Arterioscler Thromb Vasc Biol 22 (2002), 1213-1218.

[22] T. Kazumi, A. Kawaguchi, T. Hirano and G. Yoshino, Creactive protein in young, apparently healthy men: associations with serum leptin, QTc interval, and high-density lipoprotein-cholesterol, Metabolism 52 (2003), 1113-1116.

[23] S. Yaturu, R.P. Daberry, J. Rains and S. Jain, Resistin and adiponectin levels in subjects with coronary artery disease and type 2 diabetes, Cytokine 34 (2006), 21-23.

[24] W. Koeing, N. Khuseyinova, J. Baumert, C. Meisinger and H. Löwel, Serum Concentrations of Adiponectin and Risk of Type 2 Diabetes Mellitus and Coronary Heart Disease in Apparently Healthy Middle-Aged Men: Results from the 18years follow-up of a large cohort from southern Germany, JACC 48 (2006), 1369-1377.

[25] A. Wiecek, M. Adamczak and J. Chudek, Adiponectin - an adipokine with unique metabolic properties, Nephrol Dial Transplant 22 (2007), 981-988.

[26] M. von Eynatten, J.G. Schneider and P.M. Humpert, Decreased plasma lipoprotein lipase in hypoadiponectinemia: an association independent of systemic inflammation and insulin resistance, Diabetes Care 27 (2004), 2925-2929.

[27] K. Sharma, S. RamachandraRao, G. Qiu, H.K. Usui, Y. Zhu, S.R. Dunn, R. Ouedraogo, K. Hough, P. McCue, L. Chan, B. 
Falkner and B.J. Goldstein, Adiponectin regulates albuminuria and podocyte function in mice, J Clin Invest 118 (2008), 16451656.

[28] M. Barton, Reversal of proteinuric renal disease and the emerging role of endothelin, Nature Clinical Practice Nephrology 4
(2008), 490-501.

[29] C. Granier, K. Makni, L. Molina, B. Jardin-Watelet, H. Ayadi and F. Jarraya, Gene and protein markers of diabetic nephropathy, Nephrol Dial Transplant 23 (2008), 792-799. 


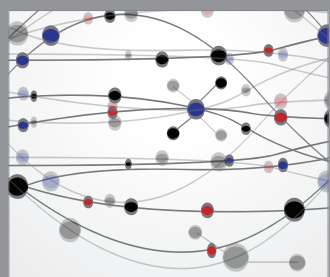

The Scientific World Journal
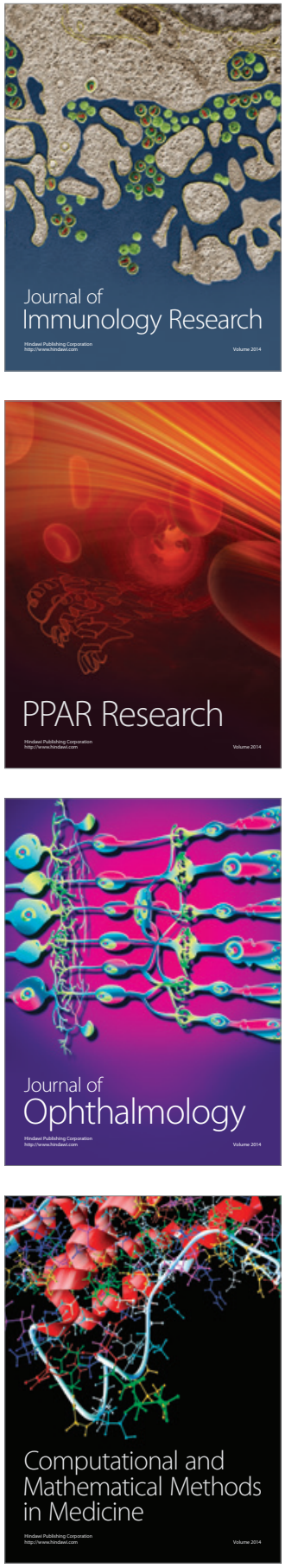

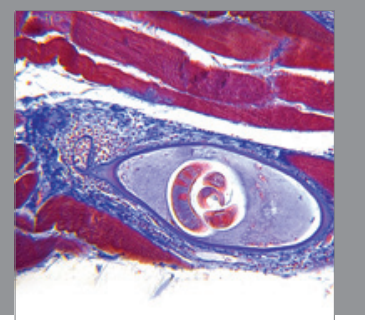

Gastroenterology

Research and Practice
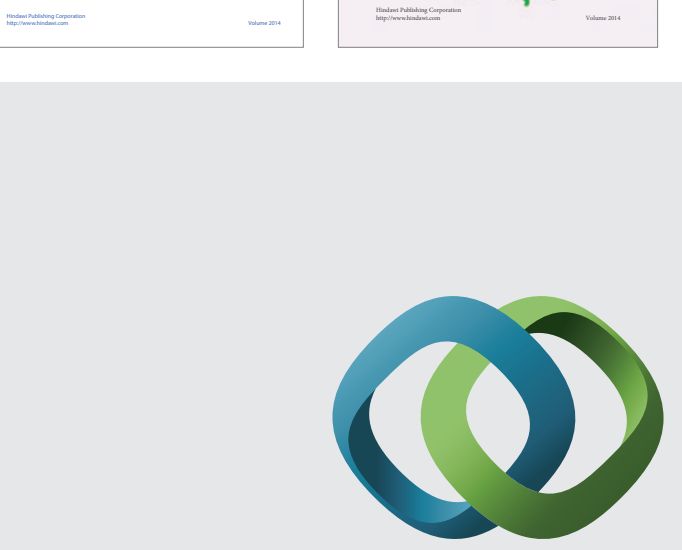

\section{Hindawi}

Submit your manuscripts at

http://www.hindawi.com
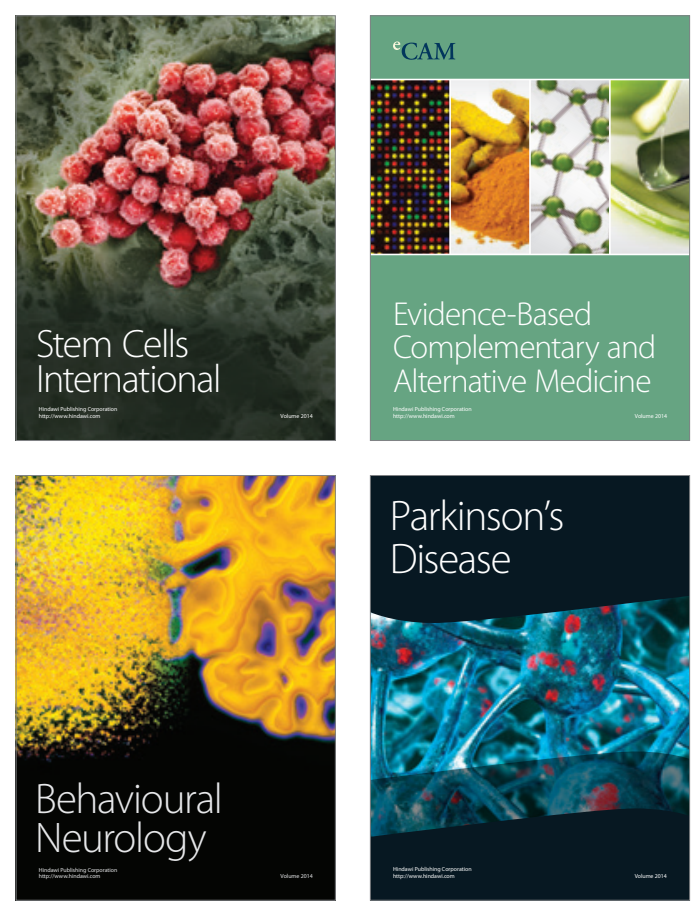

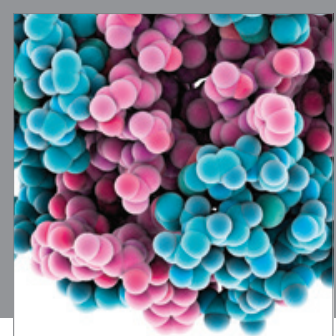

Journal of
Diabetes Research

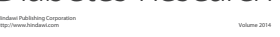

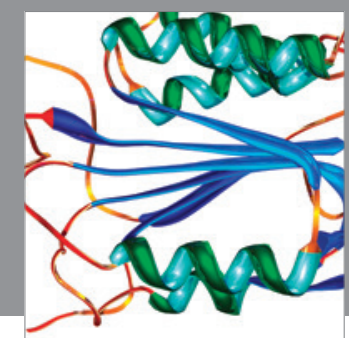

Disease Markers
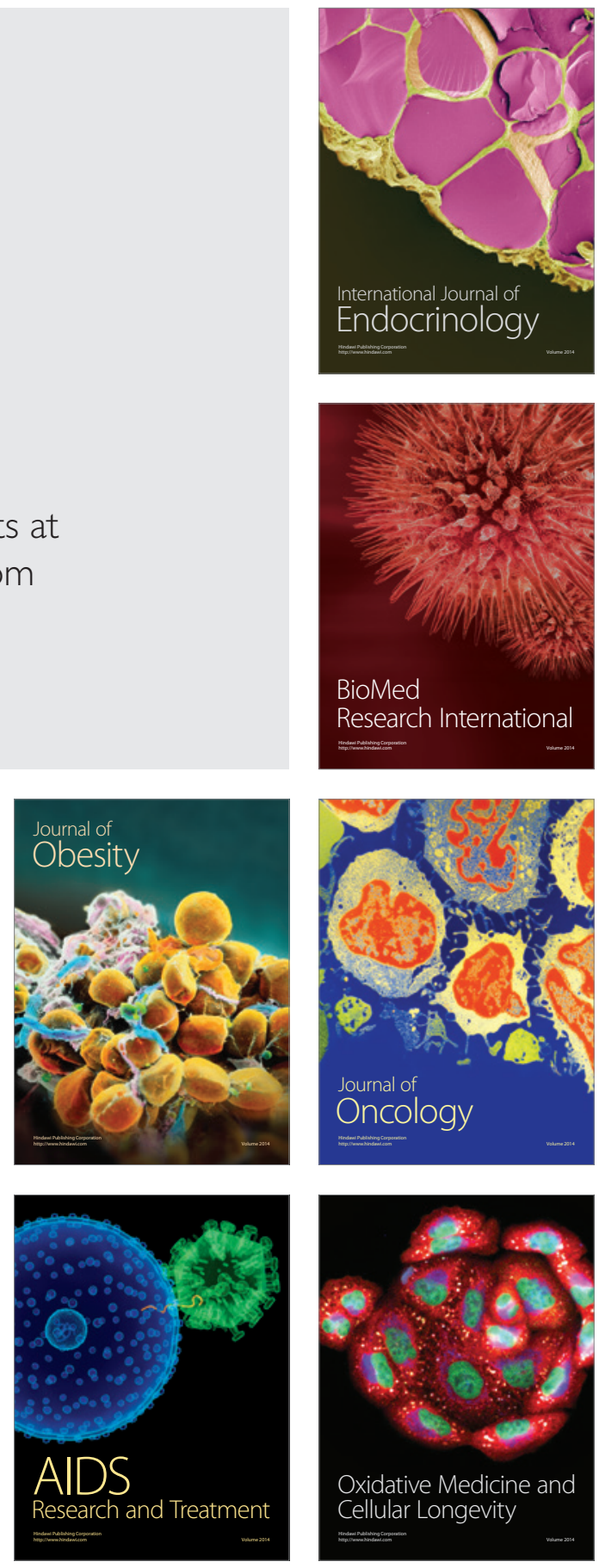